\title{
The Role of Acid Suppressants in the Prevention of Anticoagulant-Related Gastrointestinal Bleeding: A Systematic Review and Meta-Analysis
}

Chang Seok Bang ${ }^{1}$, Moon Kyung Joo ${ }^{2}$, Byung-Wook Kim³ ${ }^{3}$, Joon Sung Kim ${ }^{3}$, Chan Hyuk Park ${ }^{4}$, Ji Yong Ahn ${ }^{5}$, Jeong Hoon Lee $^{5}$, Bong Eun Lee ${ }^{6}$, Hyo-Joon Yang ${ }^{7}$, Yu Kyung $\mathrm{Cho}^{8}$, Jae Myung Park ${ }^{8}$, Beom Jin Kim ${ }^{9}$, and Hye-Kyung Jung ${ }^{10}$, Korean College of Helicobacter and Upper Gastrointestinal Research

${ }^{1}$ Department of Internal Medicine, Hallym University College of Medicine, Chuncheon, ${ }^{2}$ Division of Gastroenterology, Department of Internal Medicine, Korea University College of Medicine, ${ }^{3}$ Department of Internal Medicine, Incheon St. Mary's Hospital, College of Medicine, The Catholic University of Korea, Seoul, ${ }^{4}$ Department of Internal Medicine, Hanyang University Guri Hospital, Hanyang University College of Medicine, Guri, ${ }^{5}$ Division of Gastroenterology, Department of Internal Medicine, Asan Medical Center, University of Ulsan College of Medicine, Seoul, ${ }^{6}$ Department of Internal Medicine, Biomedical Research Institute, Pusan National University Hospital, Pusan National University School of Medicine, Busan, 'Division of Gastroenterology, Department of Internal Medicine and Gastrointestinal Cancer Center, Kangbuk Samsung Hospital, Sungkyunkwan University School of Medicine, ${ }^{8}$ Department of Internal Medicine, Seoul St. Mary's Hospital, College of Medicine, The Catholic University of Korea, ${ }^{9}$ Department of Internal Medicine, Chung-Ang University Hospital, Chung-Ang University College of Medicine, and ${ }^{10}$ Department of Internal Medicine, Ewha Medical Research Institute, Ewha Womans University School of Medicine, Seoul, Korea

Background/Aims: Although acid suppressants are widely used for the prevention or treatment of drug-induced upper gastrointestinal bleeding (GIB), evidence regarding the prevention of anticoagulant-related GIB is scarce. The aim of this study was to evaluate the protective effect of acid suppressants against anticoagulant-related GIB. Methods: A systematic review was conducted of studies that evaluated the protective effect of acid suppressants against anticoagulant-related GIB found in PubMed, the Cochrane library, Embase, and KoreaMed from the date of database inception to April 2018. Random effect model meta-analyses with sensitivity analyses were conducted. The methodological quality of each included publication was evaluated using the Risk of Bias Assessment Tool for Nonrandomized Studies. Publication bias was assessed. Results: In total, six nested case-control or cohort studies were identified and analyzed. Proton-pump inhibitors (PPI) had a protective effect against upper GIB in patients on dicumarinics (risk ratio [RR], 0.56; 95\% confidence interval [Cl], 0.38 to $0.83 ; I^{2}, 0 \%$ ); however, the histamine-2 receptor antagonist did not have the same effect (RR, 0.97; 95\% Cl, 0.52 to $1.81 ; I^{2}$, 0\%). Acid suppressants did not have a protective effect against GIB in patients on dabigatran (hazard ratio, $0.78 ; 95 \% \mathrm{Cl}, 0.44$ to $1.37 ; I^{2}$, 81.8\%). Conclusions: The protective effect of PPIs against dicumarinics-related upper GIB was clear, while there was no evidence supporting the protective effect of acid sup- pressants against dabigatran-related GIB. However, in the absence of randomized trials demonstrating a lack of bias, solid conclusions cannot be drawn. (Gut Liver 2020;14:5766)

Key Words: Anticoagulants; Acid suppressants; Gastrointestinal hemorrhage

\section{INTRODUCTION}

Anticoagulants, including dicumarinics (vitamin K antagonists; warfarin) and direct-acting oral anticoagulants (DOACs), are used to prevent or treat venous thromboembolism and to prevent arterial thromboembolism in patients with high-risk conditions, such as atrial fibrillation and valvular heart disease. ${ }^{1}$ With the aging process and increasing prevalence of cerebrocardiovascular disease in the general population, the use of anticoagulants has been increasing. ${ }^{2}$ Use of these drugs has been associated with increased risk of gastrointestinal bleeding (GIB), carrying substantial morbidity and mortality (1\% to $13 \%) .^{3-5} \mathrm{Al}-$ though DOACs have the advantage of stable bioavailability and lack of required routine serum concentration monitoring, metaanalysis showed no significant difference in the rate of GIB compared to warfarin. ${ }^{4}$

Acid suppressants, such as proton-pump inhibitors (PPIs) and histamine-2 receptor antagonists (H2RAs), have been used to

\footnotetext{
Correspondence to: Byung-Wook Kim

Division of Gastroenterology, Department of Internal Medicine, Incheon St. Mary's Hospital, College of Medicine, The Catholic University of Korea, 56 Dongsu-ro, Bupyeong-gu, Incheon 21431, Korea

Tel: +82-32-280-5051, Fax: +82-32-280-5987, E-mail: gastro@catholic.ac.kr Received on January 4, 2019. Revised on February 1, 2019. Accepted on February 13, 2019. Published online July 24, 2019. pISSN 1976-2283 eISSN 2005-1212 https://doi.org/10.5009/gnl19009

(a) This is an Open Access article distributed under the terms of the Creative Commons Attribution Non-Commercial License (http://creativecommons.org/licenses/by-nc/4.0) which permits unrestricted non-commercial use, distribution, and reproduction in any medium, provided the original work is properly cited.
} 
treat or to prevent drug-induced GIB, especially by nonsteroidal anti-inflammatory drugs (NSAIDs), including aspirin, in the upper gastrointestinal tract. ${ }^{6}$ These agents have been recommended in patients with risk factors for gastrointestinal injury, including past history of peptic ulcer or peptic ulcer complications, old age, other comorbidities, high dose-, long-term use of NSAIDs, and co-administration of corticosteroids, antiplatelet agents or anticoagulants. ${ }^{6,7}$ Although the use of anticoagulants is well known to increase the risk of GIB, data on which risk factors increase GIB and what protective measures are available are lacking. ${ }^{8}$ The aim of this study was to evaluate the protective effect of acid suppressants on anticoagulant-related GIB.

\section{MATERIALS AND METHODS}

This systematic review and meta-analysis fully adhered to the principles of the PRISMA (Preferred Reporting Items for Systematic reviews and Meta-Analyses) checklist (Supplementary Material 1). ${ }^{9}$

\section{Literature searching strategy}

PubMed, the Cochrane library, Embase, and KoreaMed were searched using common keywords associated with acid suppressants and anticoagulant-related GIB (from inception to April 2018) by two independent evaluators (C.S.B. and M.K.J.) Medical Subject Heading or Emtree keywords were selected for searching electronic databases. The abstracts of all identified studies were reviewed to exclude irrelevant publications. Fulltext reviews were performed to determine whether the inclusion criteria were satisfied in the remaining studies, and the bibliographies of relevant articles were rigorously reviewed to identify additional studies. Disagreements between the evaluators were resolved by discussion or consultation with a third evaluator (B.W.K.). The detailed searching strategy is described in Supplementary Material 2.

\section{Selection criteria}

We included studies that met the following criteria: (1) patients: patients on dicumarinics or DOACs or who were newly prescribed these agents; (2) intervention: acid suppressants administration irrespective of primary or secondary prophylaxis goal; (3) comparison: no administration of acid suppressants; (4) outcome: the incidence of GIB; (5) study design: all types including randomized, prospective or retrospective studies; (6) studies of human subjects; (7) publications in English; and (8) full-text publications. Studies that met all of the inclusion criteria were sought and selected. The exclusion criteria were as follows: (1) review articles; (2) guidelines, consensus documents or expert position papers; (3) comments, letters, brief reports, proceedings, or protocol studies; (4) case reports; (5) publications with incomplete data; and (6) meta-analysis articles. Studies meeting at least 1 of the exclusion criteria were excluded from this analysis.

\section{Methodological quality}

The methodological quality of the included publications was assessed using the Risk of Bias Assessment tool for Nonrandomized Studies (RoBANS). ${ }^{10}$ The RoBANS tool contains six domains, including the selection of participants, confounding variables, measurement of intervention (exposure), blinding of outcome assessment, incomplete outcome data, and selective outcome reporting. ${ }^{10}$ RoBANS is a validated tool that is reliable and feasible for the assessment of methodological quality of non-randomized studies. Review Manager version 5.3.3 (RevMan for Windows 7; the Nordic Cochrane Centre, Copenhagen, Denmark) was used to generate the summary of RoBANS results. Two of the evaluators (C.S.B. and M.K.J.) independently assessed the methodological qualities of all the included studies, and any disagreements between the evaluators were resolved by discussion or consultation with a third evaluator (B.W.K.).

\section{Primary and modifier-based analyses}

Two evaluators (C.S.B. and M.K.J.) independently used the same data fill-in form to collect the primary summary outcome and modifiers in each study. The outcome was the effect of acid suppressants on anticoagulant-related GIB. The common effect size extracted from each study was either risk ratios (RRs) or hazard ratios (HRs). We also performed sensitivity analyses to identify the source of heterogeneity based on the modifiers identified during the systematic review and to confirm the robustness of the main result.

\section{Handling dependence from multiple outcomes}

The independent study was the primary unit of analysis in this meta-analysis. Therefore, for the studies that reported multiple outcomes, an approach of selecting a representative single outcome to include based on the focus of the meta-analysis was used for resolving dependence. ${ }^{11,12}$

\section{Statistics}

Comprehensive Meta-Analysis Software version 3 (Biostat; Borenstein M, Hedges L, Higgins J and Rothstein H., Englewood, NJ, USA) was used for this meta-analysis. We extracted the adjusted RRs or HRs with 95\% confidence intervals (CIs) from the original articles to evaluate the effect of acid suppressants on anticoagulant-related GIB whenever possible. Heterogeneity was determined using the $I^{2}$ test developed by Higgins, which measures the percentage of total variation across studies. ${ }^{13} I^{2}$ was calculated as follows: $I^{2}(\%)=100 \times(Q-d f) / Q$, where $Q$ is Cochrane's heterogeneity statistic, and df signifies the degrees of freedom. Negative values for $I^{2}$ were set to zero, and an $I^{2}$ value over 50\% was considered to be of substantial heterogeneity (range, 0\% to 100\%). ${ }^{14}$ Pooled-effect sizes with 95\% CIs were calculated using a random effects model and the method of 
DerSimonian and Laird. ${ }^{15}$ Significance was set at $\mathrm{p}=0.05$. Publication bias was evaluated using Egger's test of the intercept and Begg and Mazumdar's rank correlation test. ${ }^{16,17}$

\section{RESULTS}

\section{Identification of relevant studies}

Fig. 1 shows how relevant studies were identified. In total, 1,597 articles were identified by the search of four databases and additional hand searching. In all, 209 were duplicate studies, and an additional 1,264 studies were excluded during the initial screening via a review of titles and abstracts. The full texts of the remaining 124 studies were then thoroughly reviewed. Among these studies, 118 articles were excluded from the final analysis. The reasons for study exclusion during the final review were as follows: narrative review article $(n=86)$, guideline, consensus document or expert position paper $(n=11)$, comment, letter, brief report, proceeding or protocol study $(n=7)$, case report $(n=7)$, incomplete data $(n=6)$, and meta-analysis $(n=1)$. The remaining 6 studies $^{5,18-22}$ were included in the qualitative synthesis.

\section{Characteristics of included studies}

In the six nested case-control or cohort studies, we identified a total of 31,645 patients (4,893 upper GIB cases vs 26,752 controls) from three nested case-control studies ${ }^{18-20}$ and 123,504 patients newly prescribed anticoagulants from three retrospective cohort studies. $^{5,21,22}$ Five studies ${ }^{5,19-22}$ performed analyses based on cohorts from a database; however, a study by Lanas et al. ${ }^{18}$ enrolled consecutive patients with upper GIB and controls in multicenter hospitals. The included studies were published between 2007 and 2016. Only one study was conducted in Asia, ${ }^{21}$ whereas the remaining studies were conducted in Europe ${ }^{18-20}$ or in the United States. ${ }^{5,22}$ The age of the enrolled population ranged from 40 to 84 years. The anticoagulants used in each study were dicumarinics in four studies ${ }^{18-20,22}$ and dabigatran in two studies ${ }^{5,21}$ (Table 1).

Although six studies were finally sought, the effect size presented by each study was different. Three nested case-control studies ${ }^{18-20}$ presented outcomes using adjusted RRs, and three retrospective cohort studies ${ }^{5,21,22}$ presented outcomes using adjusted HRs. The site and type of primary outcome of each study was also categorized by an upper GIB in three studies, ${ }^{18,20,22}$ overall GIB in two studies, ${ }^{5,21}$ and recurrent upper GIB in one study. ${ }^{19}$ The type of intervention (acid suppressants) was also categorized based on the type of acid suppressants (either PPI or H2RA) described in each study; however, the study by Lauffenburger et al. ${ }^{5}$ presented outcome values by gastrointestinal protective agents that could not be classified by subgroups (PPI or H2RA) used in the remaining studies.

Therefore, the main analysis could be categorized based on the effect size (either using HRs or RRs). The site of primary outcome of studies presenting adjusted RRs was upper GIB in two studies ${ }^{18,20}$ and recurrent upper GIB in one study ${ }^{19}$ and the type of anticoagulants of studies presenting adjusted RRs was dicumarinics in all throughout the studies. ${ }^{18-20}$ Consequently,

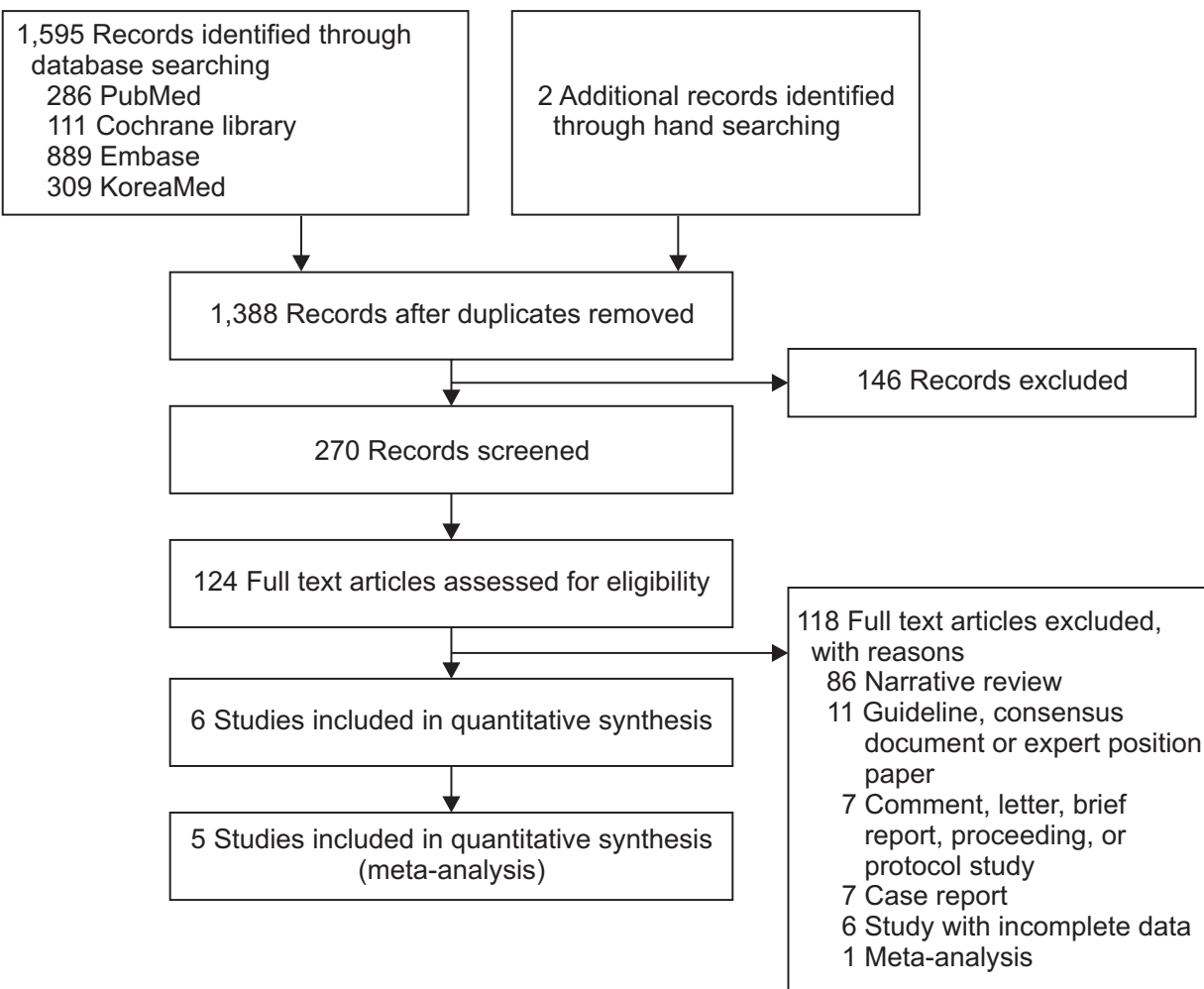

Fig. 1. Flow diagram of the identification of relevant studies. 


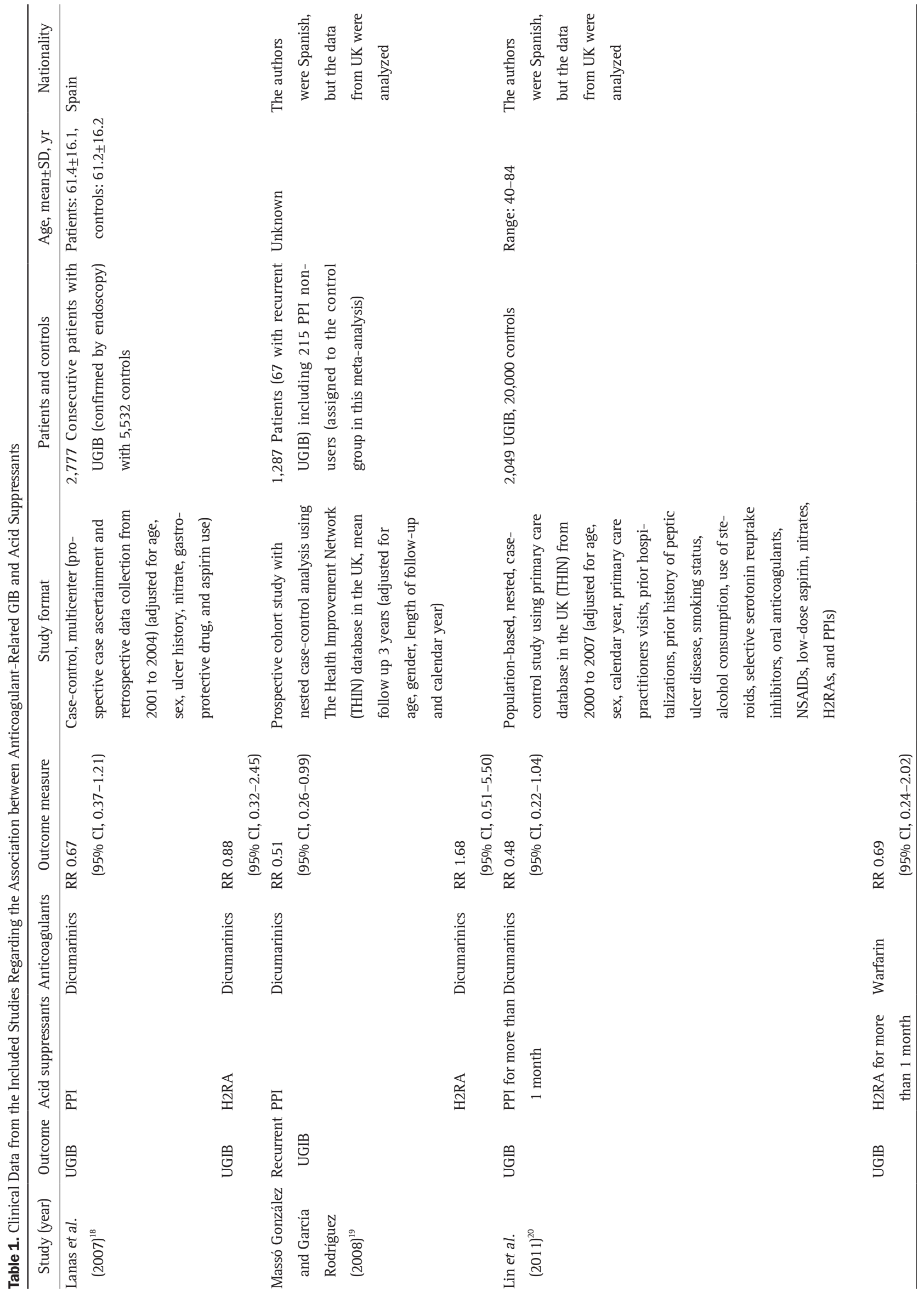



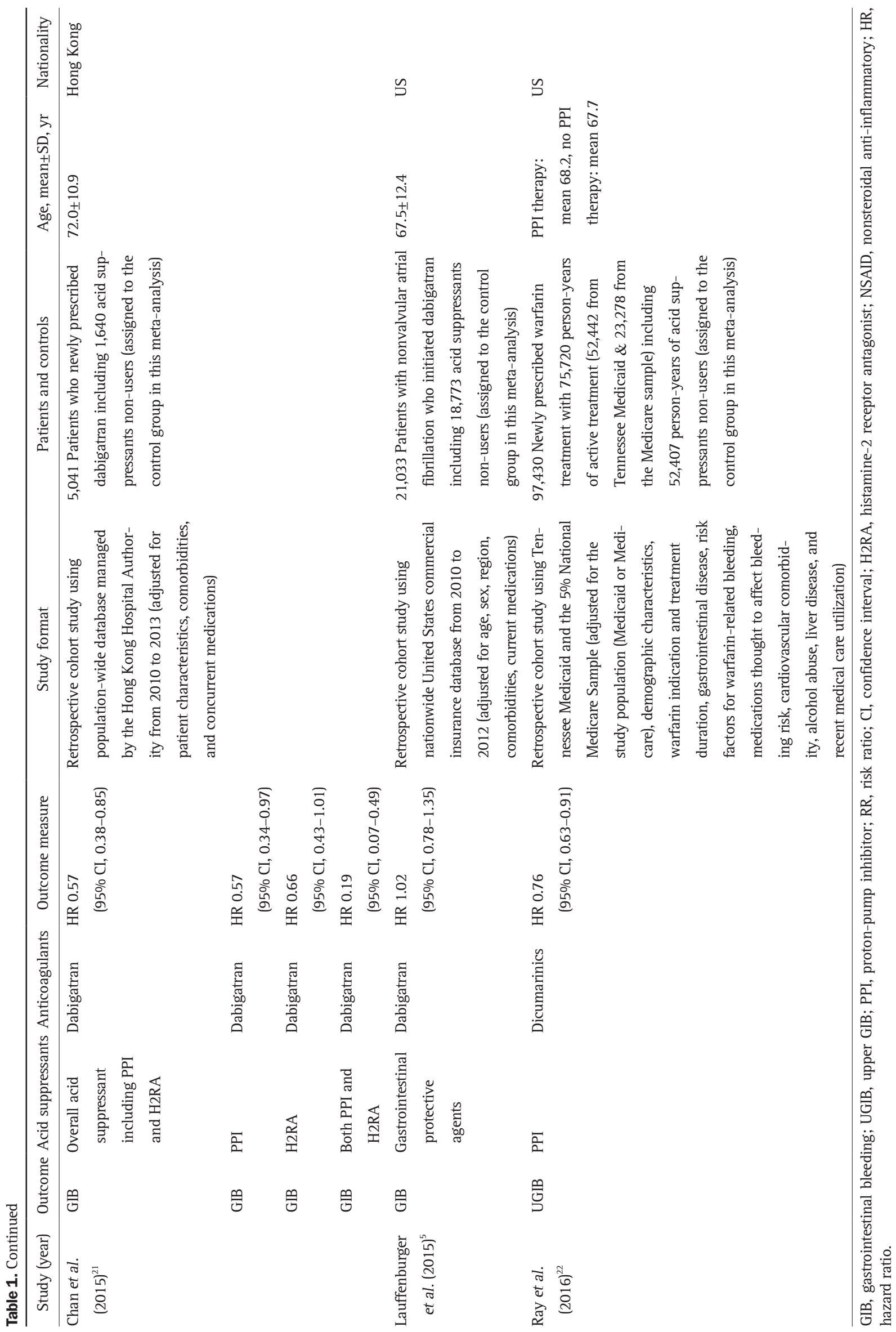
as the first-step of analysis, meta-analysis of three nested casecontrol studies using adjusted RRs reflecting the effect of PPI or H2RA on dicumarinics-related upper GIB was performed. Thereafter, sensitivity meta-analysis excluding study focused on the recurrent upper $\mathrm{GIB}^{19}$ was performed to confirm the robustness of the main analysis. Another meta-analysis of three retrospective cohort studies using adjusted HRs reflecting the effect of acid suppressants on dabigatran-related GIB was performed. Study by Ray et al. ${ }^{22}$ was included in the systematic review, however, due to the difference in effect size compared to the remaining studies (dicumarinics-associated upper GIB was the primary outcome, however, HR was the effect size, not RR), this study could not be included in the meta-analysis (Fig. 1). The clinical characteristics of the included studies are shown in Table 1.

\section{PPI or H2RA on dicumarinics-related upper GIB}

The meta-analysis of three nested case-control studies ${ }^{18-20}$ exhibited a protective effect of PPIs (RR, 0.56; 95\% CI, 0.38 to 0.83; $I^{2}, 0 \%$ ) on dicumarinics-related upper GIB (Fig. 2). However, the H2RA did not show this efficacy (RR, 0.97; 95\% CI, 0.52 to 1.81 ; $\left.I^{2}, 0 \%\right)$ (Fig. 3). There was no evidence of methodological heterogeneity.

\section{Sensitivity analysis according to the modifier}

Modifier found during systematic review was difference in type of primary outcome of each study. Only study by Massó González and García Rodríguez (2008) ${ }^{19}$ focused on the recurrent upper GIB, whereas remaining two studies in main analy$\operatorname{sis}^{18,20}$ focused on the upper GIB. Sensitivity meta-analysis of two nested case control studies ${ }^{18,20}$ excluding study by Massó González and García Rodríguez ${ }^{19}$ exhibited a consistent protective effect of PPIs (RR, 0.59; 95\% CI, 0.37 to 0.95) (Fig. 4A), and a consistent non-significant protective effect of H2RA (RR, 0.78; 95\% CI, 0.38 to 1.64) for the dicumarinics-related upper GIB (Fig. $4 \mathrm{~B})$.

\section{Acid suppressants on dabigatran-related GIB}

Two retrospective cohort studies ${ }^{5,21}$ using adjusted HRs reflecting the effect of acid suppressants on dabigatran-related GIB was performed and overall acid suppressants did not show a protective effect (HR, 0.78; 95\% CI, 0.44 to $\left.1.37 ; I^{2}, 81.8 \%\right)$ (Fig. 5).

\section{Methodological quality}

The methodological qualities of the included studies were similar, although the study by Lauffenburger et al. ${ }^{5}$ included a population with a specific disease condition of nonvalvular

\section{Study name}

Lanas A et al. (2007)

Masso Gonzalez EL et al. (2008)

Lin KJ et al. (2011)

Risk
ratio
0.670
0.510
0.480
0.564

Lower
limit
0.370
0.26
0.22
0.38

Statistics for each study Upper limit

1.212

0.995

1.044

0.829

$$
\begin{gathered}
\text { Z-value } \\
-1.325 \\
-1.974 \\
-1.852 \\
-2.916
\end{gathered}
$$

\section{Risk ratio and $95 \% \mathrm{Cl}$}

$p$-value
0.185
0.048
0.064
0.004

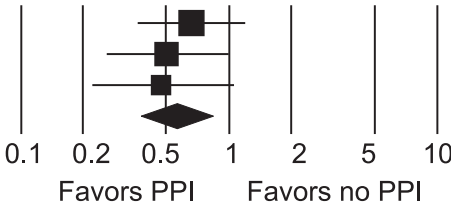

Heterogeneity: $X^{2}=0.577, \mathrm{df}=2(p=0.749) ; I^{2}=0 \%$

Test for overall effect: $Z=-2.916(p=0.004)$

Fig. 2. PPI and dicumarinics-related upper gastrointestinal bleeding. The size of each square is proportional to the study's weight. The diamond indicates the summary estimate from the pooled studies (random effect model). PPI, proton-pump inhibitor; CI, confidence interval.

Study name

Lanas A et al. (2007)

Masso Gonzalez EL et al. (2008)

Lin KJ et al. (2011)

\begin{tabular}{ccccc}
\multicolumn{5}{c}{ Statistics for each study } \\
Risk & Lower & Upper & \\
ratio & limit & limit & \multicolumn{1}{c}{ Z-value } & p-value \\
0.880 & 0.318 & 2.435 & -0.246 & 0.806 \\
1.680 & 0.512 & 5.517 & 0.855 & 0.392 \\
0.690 & 0.238 & 2.002 & -0.683 & 0.495 \\
0.968 & 0.518 & 1.809 & -0.102 & 0.918
\end{tabular}

Heterogeneity: $X^{2}=1.2480, \mathrm{df}=2(\mathrm{p}=0.536) ; I^{2}=0 \%$

Test for overall effect: $Z=-0.102(p=0.918)$

Fig. 3. H2RA and dicumarinics-related upper gastrointestinal bleeding. The size of each square is proportional to the study's weight. The diamond indicates the summary estimate from the pooled studies (random effect model).

H2RA, histamin-2 receptor antagonist; CI, confidence interval. 
A

Study name

Lanas A et al. (2007)

Lin KJ et al. (2011)

\begin{tabular}{lcccc}
\multicolumn{5}{c}{ Statistics for each study } \\
Risk & Lower & Upper & \\
ratio & limit & limit & Z-value & p-value \\
0.670 & 0.370 & 1.212 & -1.325 & 0.185 \\
0.480 & 0.221 & 1.044 & -1.852 & 0.064 \\
0.593 & 0.370 & 0.949 & -2.177 & 0.029
\end{tabular}

Risk ratio and $95 \% \mathrm{Cl}$

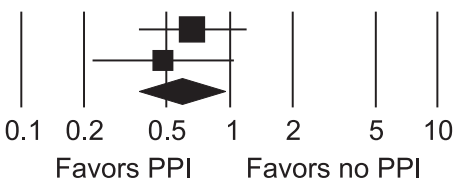

B Study name

Lanas A et al. (2007) Lin KJ et al. (2011)

\begin{tabular}{ccccc}
\multicolumn{5}{c}{ Statistics for each study } \\
Risk & Lower & Upper & \\
ratio & limit & limit & Z-value & p-value \\
0.880 & 0.318 & 2.435 & -0.246 & 0.806 \\
0.690 & 0.238 & 2.002 & -0.683 & 0.495 \\
0.784 & 0.375 & 1.635 & -0.650 & 0.516
\end{tabular}

\section{Risk ratio and $95 \% \mathrm{Cl}$}

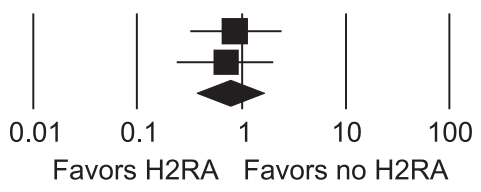

Fig. 4. Sensitivity analysis according to the modifiers. (A) PPI and dicumarinics-related upper GIB, excluding the study by Massó González EL et al. (B) H2RA and dicumarinics-related upper GIB, excluding the study by Massó González EL et al. The size of each square is proportional to the study's weight. The diamond indicates the summary estimate from the pooled studies (random effect model).

PPI, proton-pump inhibitor; GIB, gastrointestinal bleeding; H2RA, histamin-2 receptor antagonist; CI, confidence interval.

\begin{tabular}{lccccc} 
Study name & \multicolumn{5}{c}{ Statistics for each study } \\
& Hazard & Lower & Upper \\
& ratio & limit & limit & Z-value & p-value \\
Chan EW et al. (2015) & 0.570 & 0.381 & 0.852 & -2.737 & 0.006 \\
Lauffenburger JC et al. (2015) & 1.020 & 0.775 & 1.342 & 0.142 & 0.887 \\
& 0.777 & 0.440 & 1.373 & -0.867 & 0.386
\end{tabular}

Hazard ratio and $95 \% \mathrm{Cl}$

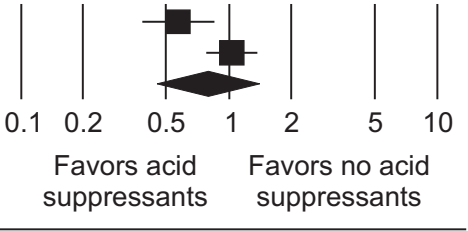

Heterogeneity: $X^{2}=5.483, \mathrm{df}=1(\mathrm{p}=0.019) ; I^{2}=81.761 \%$

Test for overall effect: $Z=-0.867(p=0.386)$

Fig. 5. Acid suppressants and dabigatran-related gastrointestinal bleeding. The size of each square is proportional to the study's weight. The diamond indicates the summary estimate from the pooled studies (random effect model).

CI, confidence interval.

atrial fibrillation compared to the remaining studies in the general population. However, subgroup analysis of methodological quality was not performed because only a small number of studies were included in this meta-analysis. The pitfalls inherent in retrospective studies make it difficult to exclude the use of over-the-counter NSAIDs, aspirin, or acid suppressants in all of the included studies. Therefore, rating for confounding variables was ranked as "unclear" risk of bias in all studies. A detailed summary of the methodological qualities of the enrolled studies is described in Fig. 6.

\section{Analysis of publication bias}

Three studies were included in the analysis of PPI or H2RA on dicumarinics-related upper GIB (Figs 2, 3) ) $^{18-20}$ and only two studies were included in the analysis of acid suppressants on dabigatran-related GIB (Fig. 5). ${ }^{5,21}$ Funnel plot or trim and fill method for the detection of publication bias was not used because of too small number of included studies. Although all the methods for the detection of publication bias were underpowered with this small number of publications, thorough statistical analyses were done in two meta-analyses (Figs 2, 3).

Egger's regression test in the analysis of PPI on dicumarinicsrelated upper GIB revealed that the intercept was -3.64 (95\% CI, -25.40 to 18.12 ), $t$-value $2.13, \mathrm{p}=0.14$ (1-tailed) and $\mathrm{p}=0.28$ (2-tailed). The rank correlation test showed that Kendall's tau was -0.67 with a continuity correction $(\mathrm{p}=0.15$ [1-tailed] and $\mathrm{p}=0.30$ [2-tailed]). Overall, there was no evidence of publication bias in this meta-analysis.

Egger's regression test in the analysis of H2RA on dicumarinics-related upper GIB revealed that the intercept was 8.37 (95\% CI, -62.44 to 79.18), $t$-value $1.50, p=0.19$ (1-tailed) and $\mathrm{p}=0.37$ (2-tailed). The rank correlation test showed that Kendall's tau 


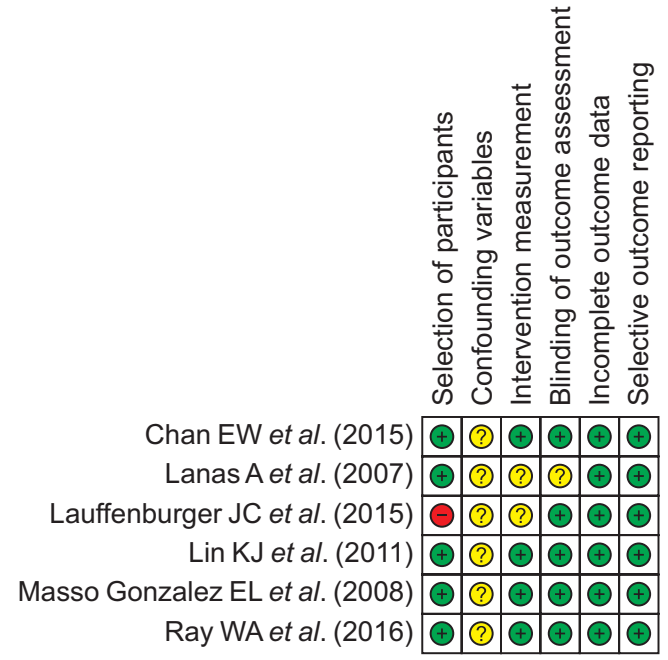

Fig. 6. RoBANS for the assessment of the methodological quality of each enrolled study (+) denotes low risk of bias, (?) denotes unclear risk of bias, (-) denotes high risk of bias.

RoBANS, Risk of Bias Assessment Tool for Nonrandomized Studies.

was 0.00 with a continuity correction $(\mathrm{p}=0.50$ [1-tailed] and p $>0.99$ [2-tailed]) Overall, there was no evidence of publication bias in this meta-analysis.

Because only two studies were included in the analysis of acid suppressants on dabigatran-related GIB, analyses for the detection of publication bias were impossible.

\section{DISCUSSION}

GIB is a major adverse event associated with the use of anticoagulants and is a frequent cause of cessation of these drugs, potentially leading to thromboembolic events. Although findings in these meta-analyses suggested the protective effect of PPIs for the development of dicumarinics-related upper GIB, this effect was attenuated by or limited in the high baseline risk of gastrointestinal injury found consistently in the enrolled studies. ${ }^{18,20-22}$ Moreover, less potent inhibition of gastric acid by H2RA showed a non-significant protective effect, suggesting that baseline ulcerogenic properties (pre-existent erosion/ulcers on the upper gastrointestinal tract, Helicobacter pylori infection, and unrecognized use of NSAIDs or aspirin) of enrolled patients determines the magnitude of the protective effect of acid suppressants on anticoagulant-related upper GIB. Recent large-scale retrospective cohort study showed consistent results with our findings. Although primary end point was the hospitalization for upper GIB, which is different from our study and published after April 2018, which could not be included in our searching strategy, PPI coadministration was associated with lower hospitalization rate for upper GIB irrelevant to the type of anticoagulants (incidence rate ratio for overall anticoagulants: 0.66, for warfarin: 0.65) (vs no PPI coadministration). ${ }^{23}$

In contrast to NSAIDs or aspirin, anticoagulants are not ul- cerogenic drugs. Pathophysiologic evidence for anticoagulants in the development of GIB is scarce. One of the suspected mechanisms is the potential for topical mucosal injury by incomplete absorption of DOACs, while warfarin is more than 95\% absorbed in the gastrointestinal tract. ${ }^{24}$ However, the increased rate of major GIB in patients taking warfarin reflects that the systemic effect of this drug is also important for the development of adverse events. ${ }^{24}$ Therefore, the exact mechanism of the protective effect of PPIs on anticoagulant-related upper GIB has not been established. Chan et al. ${ }^{21}$ presented the hypothesis that acid suppression interferes with the absorption of dabigatran (the tartaric acid core of dabigatran needs a low pH for absorption) and results in lower rates of GIB, implying a protective effect of acid suppressants on dabigatran-related GIB. However, the enrolled studies in our meta-analysis used dicumarinics and showed a protective effect of PPIs on dicumarinics-related upper GIB. Only the study by Chan et al..$^{21}$ and Lauffenburger et al. ${ }^{5}$ enrolled patients on dabigatran and the main outcome was GIB, not upper GIB in our meta-analysis. Therefore, the hypothesis by Chan et al. should be evaluated with further studies. Consequently, anticoagulants appear to potentiate GIB, especially in the upper gastrointestinal tract with pre-existent erosion/ulcers, $H$. pylori infection, and unrecognized use of NSAIDs or aspirin.

Although the results of this study revealed that co-administration of PPIs might reduce gastrointestinal bleeding, the routine use of PPIs in patients on anticoagulants should be cautious. There have been two different concerns about the overuse of PPIs in patients without risk factors and the underuse of PPIs in patients with risk factors for upper gastrointestinal injury on anticoagulant treatment. ${ }^{25,26}$ Considering that PPIs share a common metabolic enzyme (CYP3A4), implying that their use might increase the serum concentration of warfarin, some PPIs might accelerate the absorption of warfarin, and the combination of some PPIs and the CYP2C19 intermediate metabolizer could increase bleeding events. ${ }^{26-29}$ An approach that balances the risk-benefit of co-administration of PPIs and anticoagulants with estimating the individualized risk factors is needed. ${ }^{30}$ In cases with PPI and warfarin co-administration, close monitoring of prothrombin time with dose adjustment is needed. The interaction of the co-administration of PPIs and oral factor Xa inhibitors (xabans) does not appear to be of significant concern based on previous studies. ${ }^{27,31,32}$ However, the interaction of PPIs and the direct thrombin inhibitor dabigatran was reported (low on-treatment level of dabigatran) from recently published studies, ${ }^{33-35}$ and potential adverse events related to the long-term use of PPIs is another concern. Therefore, balancing the risk-benefit approach is still necessary before the co-prescription of anticoagulants and acid suppressants.

Risk factors for the development of GIB in patients taking warfarin include past history of GIB, old age, comorbidities, $H$. pylori infection, and co-administration of antiplatelet agents. ${ }^{7,8}$ However, little is known about risk factors for the development 
of GIB in patients taking DOACs. A systematic review revealed that similar risk factors consistently affected the adverse events related to dabigatran treatment, ${ }^{5}$ and these factors should be investigated using an individualized approach for the prevention of anticoagulant-related GIB.

The strength of this study was the enrollment of a large population from a balanced database reflecting real clinical practice, as it did not merely contain a group at high risk for GIB. Sensitivity analysis based on the modifier was performed, and publication bias was thoroughly investigated. Despite the strengths, several limitations were detected. First, a relatively small number of studies were enrolled in the meta-analysis, which makes it difficult to draw a solid conclusion. Second, authors could not recommend the duration or dose of PPI treatment with anticoagulants. All of the enrolled studies were nested casecontrol or cohort studies mostly from database information. Therefore, the duration, dose, and adherence to PPI could not be assessed. Third, confounding variables, including over-thecounter NSAIDs, aspirin, and antisecretory agents use, might have influenced the main results in all of the included studies. Fourth, there was no study on lower GIB, and only one study evaluated the secondary protective effect of PPIs on warfarinrelated upper GIB. ${ }^{19}$ Therefore, we could not perform subgroup analysis divided by primary or secondary protective effects of acid suppressants.

In conclusion, the protective effect of PPIs on dicumarinicsrelated upper GIB was valid and there was no evidence supporting the protective effect of acid suppressants on dabigatranrelated GIB. However, without randomized controlled trials, solid conclusions cannot be made.

\section{CONFLICTS OF INTEREST}

No potential conflict of interest relevant to this article was reported.

\section{AUTHOR CONTRIBUTIONS}

Conception and design of the study: C.S.B., B.W.K. Generation, collection, assembly, analysis and/or interpretation of data: M.K.J., B.W.K., J.S.K., C.H.P., J.Y.A., J.H.L., B.E.L., H.J.Y., Y.K.C., J.M.P., B.J.K., H.K.J. Drafting or revision of the manuscript: C.S.B. Approval of the final version of the manuscript: B.W.K.

\section{ORCID}

Chang Seok Bang

Moon Kyung Joo

Byung-Wook Kim

Joon Sung Kim

Chan Hyuk Park

Ji Yong Ahn https://orcid.org/0000-0003-4908-5431

https://orcid.org/0000-0001-6050-3695

https://orcid.org/0000-0002-2290-4954

https://orcid.org/0000-0001-9158-1012

https://orcid.org/0000-0003-3824-3481

https://orcid.org/0000-0002-0030-3744
Jeong Hoon Lee

Bong Eun Lee

Hyo-Joon Yang

Yu Kyung Cho

Beom Jin Kim

Hye-Kyung Jung
Jae Myung Park https://orcid.org/0000-0002-0778-7585

https://orcid.org/0000-0003-2734-2134

https://orcid.org/0000-0002-0265-672X https://orcid.org/0000-0002-7297-6577 https://orcid.org/0000-0002-1534-7467 https://orcid.org/0000-0002-6653-5214 https://orcid.org/0000-0002-0938-6697

\section{REFERENCES}

1. Weitz JI, Eikelboom JW, Samama MM. New antithrombotic drugs: antithrombotic therapy and prevention of thrombosis, 9th ed. American College of Chest Physicians Evidence-Based Clinical Practice Guidelines. Chest 2012;141(2 Suppl):e120S-e151S.

2. Barnes GD, Lucas E, Alexander GC, Goldberger ZD. National trends in ambulatory oral anticoagulant use. Am J Med 2015;128:13001305.

3. Coleman CI, Sobieraj DM, Winkler S, et al. Effect of pharmacological therapies for stroke prevention on major gastrointestinal bleeding in patients with atrial fibrillation. Int J Clin Pract 2012;66:5363.

4. Miller CS, Dorreen A, Martel M, Huynh T, Barkun AN. Risk of gastrointestinal bleeding in patients taking non-vitamin $\mathrm{K}$ antagonist oral anticoagulants: a systematic review and meta-analysis. Clin Gastroenterol Hepatol 2017;15:1674-1683.

5. Lauffenburger JC, Rhoney DH, Farley JF, Gehi AK, Fang G. Predictors of gastrointestinal bleeding among patients with atrial fibrillation after initiating dabigatran therapy. Pharmacotherapy 2015;35:560-568.

6. Abraham NS, Hlatky MA, Antman EM, et al. ACCF/ACG/AHA 2010 expert consensus document on the concomitant use of proton pump inhibitors and thienopyridines: a focused update of the ACCF/ACG/AHA 2008 expert consensus document on reducing the gastrointestinal risks of antiplatelet therapy and NSAID use. Am J Gastroenterol 2010;105:2533-2549.

7. Hernández-Díaz S, Rodríguez LA. Association between nonsteroidal anti-inflammatory drugs and upper gastrointestinal tract bleeding/perforation: an overview of epidemiologic studies published in the 1990s. Arch Intern Med 2000;160:2093-2099.

8. Barada K, Abdul-Baki H, El Hajj II, Hashash JG, Green PH. Gastrointestinal bleeding in the setting of anticoagulation and antiplatelet therapy. J Clin Gastroenterol 2009;43:5-12.

9. Moher D, Liberati A, Tetzlaff J, Altman DG; PRISMA Group. Preferred reporting items for systematic reviews and meta-analyses: the PRISMA statement. Ann Intern Med 2009;151:264-269.

10. Kim SY, Park JE, Lee YJ, et al. Testing a tool for assessing the risk of bias for nonrandomized studies showed moderate reliability and promising validity. J Clin Epidemiol 2013;66:408-414.

11. Card N. Applied meta-analysis for social science research. New York: The Guilford Press, 2012.

12. Higgins JP, Green S. Cochrane handbook for systematic reviews of interventions. Version 5.1.0. London: The Cochrane Collaboration, 
2011.

13. Higgins JP, Thompson SG. Quantifying heterogeneity in a metaanalysis. Stat Med 2002;21:1539-1558.

14. Higgins JP, Thompson SG, Deeks JJ, Altman DG. Measuring inconsistency in meta-analyses. BMJ 2003;327:557-560.

15. DerSimonian R, Laird N. Meta-analysis in clinical trials. Control Clin Trials 1986;7:177-188.

16. Begg CB, Mazumdar M. Operating characteristics of a rank correlation test for publication bias. Biometrics 1994;50:1088-1101.

17. Egger M, Davey Smith G, Schneider M, Minder C. Bias in metaanalysis detected by a simple, graphical test. BMJ 1997;315:629634.

18. Lanas A, García-Rodríguez LA, Arroyo MT, et al. Effect of antisecretory drugs and nitrates on the risk of ulcer bleeding associated with nonsteroidal anti-inflammatory drugs, antiplatelet agents, and anticoagulants. Am J Gastroenterol 2007;102:507-515.

19. Massó González EL, García Rodríguez LA. Proton pump inhibitors reduce the long-term risk of recurrent upper gastrointestinal bleeding: an observational study. Aliment Pharmacol Ther 2008;28:629-637.

20. Lin KJ, Hernández-Díaz S, García Rodríguez LA. Acid suppressants reduce risk of gastrointestinal bleeding in patients on antithrombotic or anti-inflammatory therapy. Gastroenterology 2011;141:71-79.

21. Chan EW, Lau WC, Leung WK, et al. Prevention of dabigatranrelated gastrointestinal bleeding with gastroprotective agents: a population-based study. Gastroenterology 2015;149:586-595.

22. Ray WA, Chung CP, Murray KT, et al. Association of proton pump inhibitors with reduced risk of warfarin-related serious upper gastrointestinal bleeding. Gastroenterology 2016;151:1105-1112.

23. Ray WA, Chung CP, Murray KT, et al. Association of oral anticoagulants and proton pump inhibitor cotherapy with hospitalization for upper gastrointestinal tract bleeding. JAMA 2018;320:22212230.

24. Di Minno A, Spadarella G, Prisco D, Scalera A, Ricciardi E, Di Minno G. Antithrombotic drugs, patient characteristics, and gastrointestinal bleeding: clinical translation and areas of research.
Blood Rev 2015;29:335-343.

25. Savarino V, Dulbecco P, de Bortoli N, Ottonello A, Savarino E. The appropriate use of proton pump inhibitors (PPIs): need for a reappraisal. Eur J Intern Med 2017;37:19-24.

26. Sugano K. How do we manage serious gastrointestinal adverse events associated with anti-thrombotic therapy? Expert Rev Gastroenterol Hepatol 2015;9:5-8.

27. Agewall S, Cattaneo M, Collet JP, et al. Expert position paper on the use of proton pump inhibitors in patients with cardiovascular disease and antithrombotic therapy. Eur Heart J 2013;34:17081713.

28. Teichert M, van Noord C, Uitterlinden AG, et al. Proton pump inhibitors and the risk of overanticoagulation during acenocoumarol maintenance treatment. Br J Haematol 2011;153:379-385.

29. Hata M, Shiono M, Akiyama K, et al. Incidence of drug interaction when using proton pump inhibitor and warfarin according to cytochrome P450 2C19 (CYP2C19) genotype in Japanese. Thorac Cardiovasc Surg 2015;63:45-50.

30. Ko D, Hylek EM. Anticoagulation in the older adult: optimizing benefit and reducing risk. Semin Thromb Hemost 2014;40:688694.

31. EINSTEIN Investigators, Bauersachs R, Berkowitz SD, et al. Oral rivaroxaban for symptomatic venous thromboembolism. N Engl J Med 2010;363:2499-2510.

32. Granger CB, Alexander JH, McMurray JJ, et al. Apixaban versus warfarin in patients with atrial fibrillation. N Engl J Med 2011;365:981-992.

33. Kuwayama T, Osanai H, Ajioka M, et al. Influence of proton pump inhibitors on blood dabigatran concentrations in Japanese patients with non-valvular atrial fibrillation. J Arrhythm 2017;33:619-623.

34. Bolek T, Samoš M, Stančiaková L, et al. The impact of proton pump inhibition on dabigatran levels in patients with atrial fibrillation. Am J Ther 2019;26:e308-e313.

35. Bolek T, Samoš M, Škorňová I, et al. Proton pump inhibition in patients treated with novel antithrombotic drugs: should we worry about thrombosis? J Cardiovasc Pharmacol 2018;72:71-76. 